\title{
Acute hypoxemia due to lung collapse in COVID-19: the role of therapeutic bronchoscopy
}

\begin{abstract}
Bronchoscopy is an aerosol-generating procedure and involves a high risk of transmission of SARS-CoV-2 to health care workers. There are very few indications for performing bronchoscopy in a patient with confirmed COVID-19. These include atelectasis, foreign body aspiration, and suspected superinfection in immunocompromised patients. Proper use of standard personal protective equipment is mandatory to reduce the risk of transmission to health care workers. In this article, we describe a case of acute lung collapse in a 16-year-old boy with cerebral palsy who was infected with COVID-19. This patient responded to therapeutic bronchoscopy and had complete resolution of lung collapse within 24 hours of the procedure.
\end{abstract}

Key words: bronchoscopy, SARS-CoV-2, COVID-19, atelectasis

Adv Respir Med. 2021; 89: 448-450

\section{Introduction}

Therapeutic bronchoscopy is frequently used in the intensive care unit (ICU) due to lobar atelectasis that is unresponsive to chest physiotherapy [1]. It is an aerosol-generating procedure that has a high risk of transmission of coronavirus disease 2019 (COVID-19) to health care workers (HCWs). Specific guidelines have been introduced for performing bronchoscopy during the time of the COVID-19 pandemic, and these guidelines include instructions on the use of adequate personal protective equipment (PPE) [2-5]. Bronchoscopy in confirmed COVID-19 patients is indicated in conditions such as lung collapse due to mucus plugging or foreign body aspiration, suspected superinfections in immunocompromised patients, management of massive hemoptysis (in conjunction with other measures), central airway obstruction, bronchoscopic intubation, and percutaneous tracheostomy [3]. However, the role of bronchoscopy is limited in the diagnosis of COVID-19 due to the substantial risk of transmission of the disease to health care workers, and because of the availability of low- risk and non-invasive upper respiratory samples obtained from oropharyngeal and nasopharyngeal swabs. Here, we present a case of a young male who presented with acute worsening hypoxemia and left lung collapse with a history of contact with a confirmed COVID-19 patient who was managed with therapeutic bronchoscopy.

\section{Case presentation}

A 16-year-old boy presented to the emergency department with a history of progressive shortness of breath for three days. There was a doubtful history of aspiration of a piece of a toy (in the form of a wool ball) three days ago during an episode of a seizure. There was no history of upper respiratory symptoms or fever. The patient was previously diagnosed with cerebral palsy with spastic diplegia, and also had a seizure disorder. He had been bedridden for three years and was dependant for all activities of daily living. His mother was the primary caretaker and was detected to be COVID-19 positive about two weeks back, but was asymptomatic. On examination, he had tachypnea with a respiratory rate of

Address for correspondence: Saurabh Mittal, Department of Pulmonary, Critical Care and Sleep Medicine, All India Institute of Medical Sciences (AllMS), New Delhi, India,

e-mail: saurabh kgmu@yahoo.co.in

DOI: 10.5603/ARM..a2021.0009

Received: 04.10 .2020

Copyright (C) 2021 PTChP

ISSN 2451-4934 

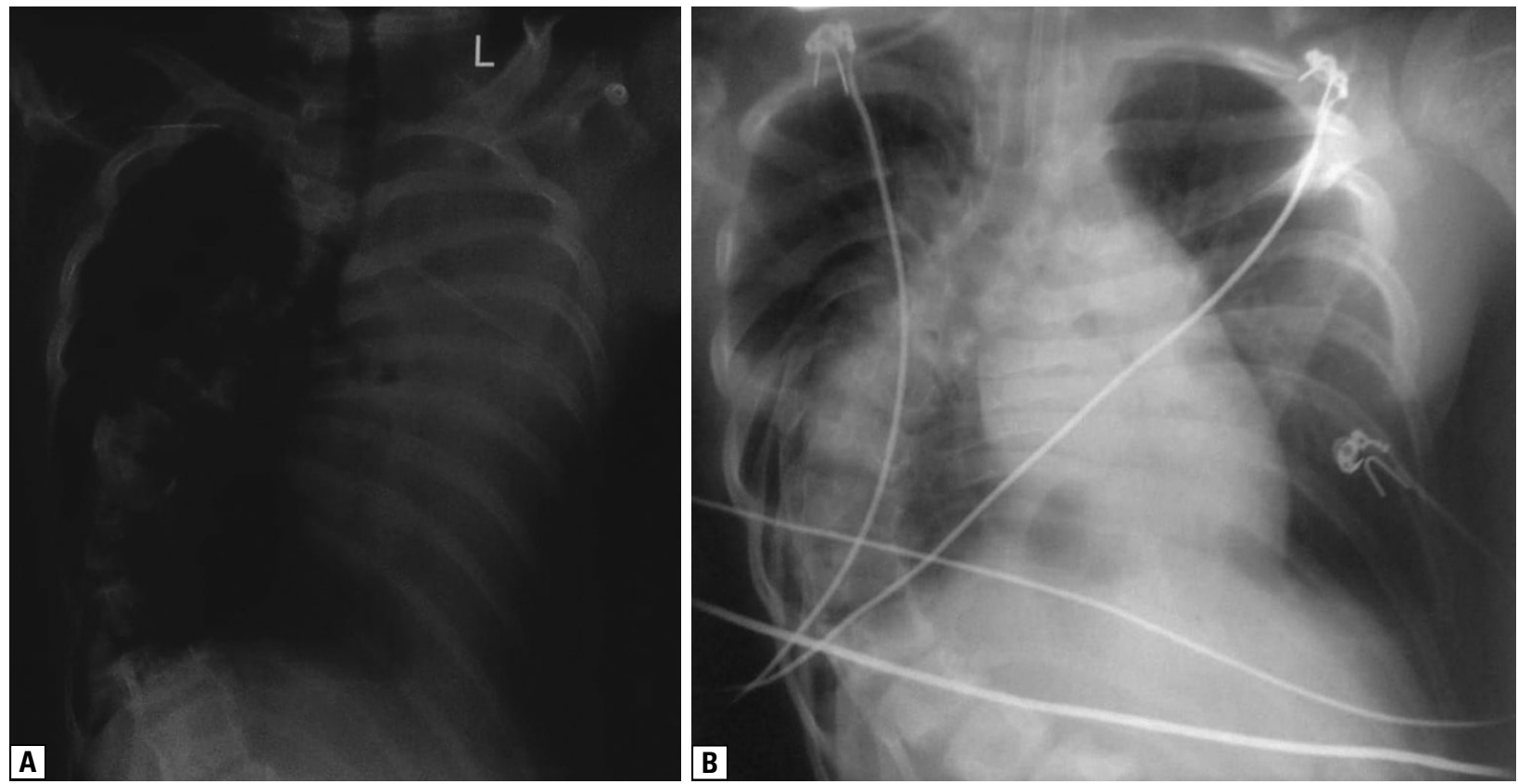

Figure 1. A. The chest radiograph demonstrating scoliosis with complete opacification of the left hemithorax with a mediastinal shift to the same side suggesting left lung collapse; B. The chest radiograph following bronchoscopy demonstrating complete clearance of the left lung with aeration

30 per minute, and a saturation of $95 \%$ on oxygen inhalation at $8 \mathrm{~L} / \mathrm{min}$ with a facemask. He was hemodynamically stable. His nasopharyngeal and oropharyngeal swabs were sent for COVID-19 RTPCR and came back positive. Chest X-ray demonstrated left lung collapse with scoliosis (Figure 1A). A contrast-enhanced computed tomography was ordered because of the suspicion for foreign body aspiration. It revealed an oval, smooth lesion in the distal left main bronchus which was suspicious for a foreign body or mucous plugging. There was also evidence of a collapsed left upper and lower lobe. There was a gross scoliotic deformity with convexity towards the right and a resultant deviation of the mediastinum and vascular structures to the left hemithorax. He was transferred to the ICU as a result of an increased need for oxygen. Due to worsening hypoxemia, a decision to begin therapeutic bronchoscopy was undertaken. For the procedure, all the necessary equipment and materials were prepared outside the COVID-19 ICU. This included saline, syringes, and the bronchoscopy system (therapeutic bronchoscope with a monitor). A negative pressure room was not available for the procedure. As per recommendations, all PPE were used. These included an N95 mask, goggles, two sets of gloves, and a plastic protective gown which included a head and neck cover. Flexible bronchoscopy was performed via the oral route under sedation. The sedative agents used included propofol and fen- tanyl. Topical anesthesia via oropharyngeal spray and the 'bronchoscopic spray as you go' method were provided using $2 \%$ lidocaine. The left main bronchus showed a large thick mucus plug which was removed with thorough suctioning following saline instillation. No foreign body could be identified. Following the procedure, the patient required intubation due to worsening hypoxia. A chest X-ray performed 24-hours following the procedure showed complete expansion of the left lung (Figure 1B). He was further managed using standard COVID-19 care protocols which included administration of steroids and hydroxychloroquine. He was subsequently extubated on day three of admission and was discharged home on day 12 with a room air oxygen saturation of $97 \%$.

\section{Discussion}

This case describes the therapeutic role of bronchoscopy in a patient with confirmed COVID-19. The use of therapeutic bronchoscopy in this patient facilitated his recovery from acute hypoxemic respiratory failure.

Across numerous studies, flexible bronchoscopy has been shown to be effective in removing secretions and improving atelectasis with success rates between $79-89 \%$ [6]. Lobar atelectasis responds better than subsegmental atelectasis. This is likely due to the larger size of mucus plugs which are amenable for bronchoscopic removal. 
Other modalities for the treatment of atelectasis (i.e. chest physiotherapy) are also useful in the majority of individuals. A randomized controlled trial studying the use of an aggressive chest physiotherapy regimen versus the use of bronchoalveolar lavage for the treatment of acute atelectasis showed the same extent of improvement at 24 and 48 hours in terms of the radiological resolution of the atelectasis [6]. Patients with restrictive lung diseases, such as neuromuscular diseases, are more prone to develop atelectasis due to mucous plugging. If these patients develop pneumonia, lobar or complete collapse of the lung can occur. In such conditions, bronchoscopy remains the procedure of choice for its diagnostic and therapeutic value. This was affirmatively the case in our patient as there was suspicion of an aspirated foreign body and an inability to cooperate with aggressive chest physiotherapy due to his severe hypoxia and his comorbid cerebral palsy. As a result, we decided to treat the patient with therapeutic bronchoscopy. Bronchoscopy, in critically ill patients, is not without risk. The overall incidence of complications and mortality during fiberoptic bronchoscopy based on a large prospective study covering 19 Italian centres encompassing 20,986 bronchoscopies was approximately between $0.02 \%$ and $1.1 \%$ [7]. The most common complications of bronchoscopy are bronchospasm, hypoxemia, cardiac arrhythmias, hypotension, bleeding and hemoptysis, pneumothorax, myocardial infarction/pulmonary edema, and death [8]. Our patient also needed intubation in the post-procedural period due to hypoxia. His oxygen requirement prior to the procedure was high. We initially planned to electively intubate him before bronchoscopy. However, keeping in mind the poor prognosis of COVID-19 patients on invasive mechanical ventilation, and after discussion with family members, a decision for proceeding with bronchoscopy was made.

A previous study described 101 bronchoscopies in 93 patients with COVID-19 on invasive mechanical ventilation with the major indication being superinfection (63/101), followed by airway secretion management with or without atelectasis (38/101) [9]. Ninety-five per cent of the patients showed thick white gelatinous secretions that were difficult to suction. This could be the result of either direct viral effect or because of heat moisture exchanger use among ventilated patients. One bronchoscopist out of the three performing the procedure was infected, but it is unclear whether the infection was procedure-related. In our patient, none of the healthcare workers involved in bronchoscopy were diagnosed with COVID-19 during the four week period following bronchoscopy.

\section{Conclusion}

In cases with definitive indications, therapeutic bronchoscopy should not be delayed in COVID-19 confirmed cases as the procedure may be lifesaving. Proper use of PPE helps in reducing the risk of transmission of the disease to health care workers.

\section{Conflict of interest}

None declared.

\section{References:}

1. Taylor DL. Bronchoscopy: What critical care nurses need to know. Crit Care Nurs Clin North Am. 2010; 22(1): 33-40, doi: 10.1016/j.ccell.2009.10.004, indexed in Pubmed: 20193878.

2. Luo F, Darwiche K, Singh S, et al. Performing bronchoscopy in times of the COVID-19 pandemic: practice statement from an international expert panel. Respiration. 2020; 99(5): 417-422, doi: 10.1159/000507898, indexed in Pubmed: 32344422.

3. Cordovilla R, Álvarez S, Llanos L, et al. Recomendaciones de consenso SEPAR y AEER sobre el uso de la broncoscopia y la toma de muestras de la vía respiratoria en pacientes con sospecha o con infección confirmada por COVID-19. Archivos de Bronconeumología. 2020; 56: 19-26, doi: 10.1016/j. arbres.2020.03.017.

4. Wahidi MM, Shojaee S, Lamb CR, et al. The use of bronchoscopy during the coronavirus disease 2019 pandemic: CHEST/ AABIP Guideline and Expert Panel Report. Chest. 2020; 158(3): 1268-1281, doi: 10.1016/j.chest.2020.04.036, indexed in Pubmed: 32361152.

5. Guedes F, Boléo-Tomé JP, Rodrigues LV, et al. Recommendations for interventional pulmonology during COVID-19 outbreak: a consensus statement from the Portuguese Pulmonology Society. Pulmonology. 2020; 26(6): 386-397, doi: 10.1016/j.pulmoe.2020.07.007, indexed in Pubmed: 32868252.

6. Kreider ME, Lipson DA. Bronchoscopy for atelectasis in the ICU: a case report and review of the literature. Chest. 2003; 124(1): 344-350, doi: 10.1378/chest.124.1.344, indexed in Pubmed: 12853543.

7. Ergan B, Nava S. The use of bronchoscopy in critically ill patients: considerations and complications. Expert Rev Respir Med. 2018; 12(8): 651-663, doi: 10.1080/17476348.2018.1494576, indexed in Pubmed: 29958019.

8. Mohan A, Madan K, Hadda V, et al. Jayachandra. Guidelines for diagnostic flexible bronchoscopy in adults: Joint Indian Chest Society/National College of chest physicians (I)/Indian association for bronchology recommendations. Lung India. 2019; 36(Supplement): S37-S89, doi: 10.4103/lungindia.lungindia_108_19, indexed in Pubmed: 32445309.

9. Torrego A, Pajares V, Fernández-Arias C, et al. Bronchoscopy in patients with COVID-19 with invasive mechanical ventilation: a single-center experience. Am J Respir Crit Care Med. 2020; 202(2): 284-287, doi: 10.1164/rccm.202004-0945LE, indexed in Pubmed: 32412787. 